\title{
Application of Lock-in Amplifier to Weak Magnetic Field Detection
}

\author{
Hengli Song \\ College of Automation \\ China University of Geosciences \\ Wuhan, China \\ e-mail: songhengli@163.com
}

\author{
Haobin Dong, Lin Zhang \\ College of Automation \\ China University of Geosciences \\ Wuhan, China \\ e-mail: donghb@cug.edu.cn, zhanglin_cug@163.com
}

\begin{abstract}
A lock-in amplifier (LIA) is designed and optimized to recover weak magnetic signals from noise. The proposed LIA is based on phase-sensitive detection technique (PSD), obtain needed signal at the specific reference frequency and reject noise signals at other frequencies. A measurement system applies this LIA to operate at a particular reference frequency which avoids interferences. The application of the LIA is the weak magnetic signal detection in a high noise level environment. The desired signal is in voice frequency. In this system the LIA acts as a detector and programmable low pass filter combined. Experiments are performed to verify the feasibility of the LIA. The result of the test shows that very small signals can be detected in the presence of large amounts of uncorrelated noise when the frequency and phase of the desired signal are known.
\end{abstract}

Keywords- LIA; Weak Signal Detection; PSD; Magnetic Field; LPF

\section{INTRODUCTION}

Measurement of weak magnetic signal plays a vital role in various scientific fields. However, accurate detection of weak signal in a high noise level environment is always difficult, especially in the case that the output signal of magnetic sensor is smaller than the noise level. Numbers of methods to solve the problem have been researched in literatures. All these methods such as wavelet analysis[1], fuzzy detection[2],time-frequency analysis[3] and stochastic resonance[4] are senior to detect special signals obscured by noise and interference signals but not realizable in hardware due to their complicacy. One the other hand, conventional methods, such as linear filtering, are evidently not effective on extracting the signal information[5, 6]. Therefore, special amplification techniques have to be tackled to increase the signal-tonoise ratio (SNA) of measurement system. One of the most possibilities is lock-in amplifiers (LIAs) which are widely used in recovering weak signals from noise[7]. LIAs, based on the phase-sensitive detection technique (PSD), obtain needed signal at the specific reference frequency and reject noise signals at other frequencies. Reference[8] designed an analogue lock-in amplifier supplied by single $3 \mathrm{~V}$ power source for processing output sensor signals in highly noisy situations. Reference[9] also presents an analog LIA with single-supply voltage for battery-operated applications. The LIA is suitable for its minimum size and low power. Reference[10] combines a lock-in detection scheme with pulsed electrically detected magnetic resonance signal modulation to reduce the low-frequency noise level. Reference[11] concentrates on the application of fully-analog lock-in amplifier to the accurate measurements of gas. The designed circuit has been optimized to operate at a particular reference frequency which avoids also any kind of interferences at $50 \mathrm{~Hz}$ net frequency and its harmonics.

In this paper, we focus on the weak magnetic signal detection in a high noise level environment. A LIA is proposed to detect voice frequency signal. The lock-in amplifiers acts as a detector and programmable low pass filter combined. Very small signals can be detected in the presence of large amounts of uncorrelated noise when the frequency and phase of the desired signal are known. Then we will introduce the basic principle of an induced magnetometer and its framework. And we perform an experiment to verify the feasibility of the proposed LIA. Finally, some conclusions are drawn.

\section{DESIGN OF LIA CIRCUIT}

\section{A. Lock-In Amplifier Architecture}

We use a LIA to detect the signal based on a correlation theory. Fig .1 shows the block diagram of the LIA. It consists of two parallel phase sensitive detector (PSD) branches the aim of which is to remove the phase dependence of system.

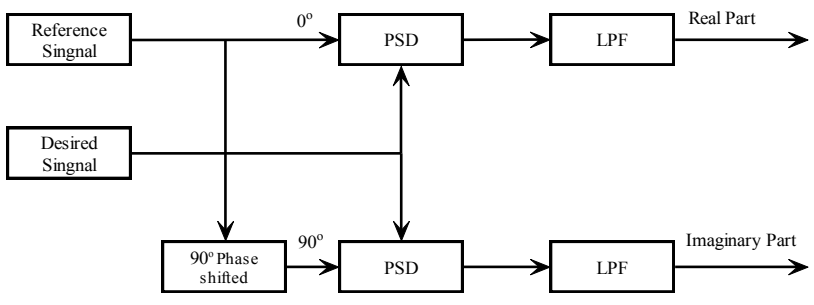

Figure 1. Lock-in amplifier architecture

The output of the each PSD is the multiplication of its two input signals one of which is the detected signal while the other is the reference square wave signal or $90^{\circ}$ phaseshifted square wave signal.

A sensor is triggered by the reference signal with a known frequency $f_{0}$ and its response $V_{s}$ is injected into the PSD.

Suppose the desired signal is as follow.

$$
x(t)=V_{s} \cos \left(\omega_{0} t+\theta\right)
$$


Where $\omega_{0}=2 \pi f_{0}$ is the angular frequency of the detected signal. $\theta$ is the initial phase of the detected signal. $t$ is a variate of time.

The other input of the PSD is a square wave which is described as follow.

$$
r(t)=a_{0}+\sum_{m=1}^{\infty} a_{m} \cos m \omega_{0} t+\sum_{m=1}^{\infty} b_{m} \sin m \omega_{0} t
$$

Where the is $a_{0}$ the DC component, $a_{m}$ is the Fourier coefficient of cosine components, $b_{m}$ is the Fourier coefficient of sine components.

The PSD multiplies the input signal by a square reference signal, denoted by $V_{\text {ref }}$.

Thus the Fourier series of the $r(t)$ is as follow.

$$
r(t)=\frac{4 V_{s}}{\pi} \sum_{n=1}^{\infty} \frac{(-1)^{n+1}}{2 n-1} \cos \left[(2 n-1) \omega_{0} t\right]
$$

The output of the PSD is as follow.

$$
\begin{aligned}
& u_{P S D}(t)=x(t) r(t) \\
& =V_{s} \cos \left(\omega_{0} t+\theta\right) \times \frac{4 V_{s}}{\pi} \sum_{n=1}^{\infty} \frac{(-1)^{n+1}}{2 n-1} \cos \left[(2 n-1) \omega_{0} t\right] \\
& =\frac{2 V_{s} V_{r e f}}{\pi} \sum_{n=1}^{\infty} \frac{(-1)^{n+1}}{2 n-1} \cos \left[(2 n-2) \omega_{0} t-\theta\right] \\
& +\frac{2 V_{s} V_{r e f}}{\pi} \sum_{n=1}^{\infty} \frac{(-1)^{n+1}}{2 n-1} \cos \left[2 n \omega_{0} t+\theta\right]
\end{aligned}
$$

After a low-pass filter (LPF) with a suitable low cut-off frequency, the desired signal's DC component may be easily extracted. The output is given by the following,

$$
u_{\text {LIA } 0}=\frac{2 V_{s} V_{r e f}}{\pi} \cos \theta
$$

Equation(5) implies the real component of the detected signal with the $0^{\circ}$ phase-shifted square wave reference signal. If the reference signal is $90^{\circ}$ phase-shifted square wave, the output is given by the following,

$$
u_{\text {LIA90 }}=\frac{2 V_{s} V_{\text {ref }}}{\pi} \sin \theta
$$

Equation(6) implies the imaginary part. From the above equations (5) and (6), the amplitude and the phase can be calculated as follow.

$$
\left\{\begin{array}{l}
V_{s}=\frac{\pi}{2 V_{\text {ref }}} \sqrt{{u_{\text {LIA0 }}{ }^{2}+u_{\text {LIA90 }}^{2}}^{2}} \\
\theta=\arctan \frac{u_{\text {LIA } 90}}{u_{\text {LIA } 0}}
\end{array}\right.
$$

\section{B. Square Wave Generator and Phase-shifting Circuit}

The references of the two PSD branches are square wave generator and phase-shifting circuits. Direct Digital Synthesizer (DDS) AD9851 is convenient to accomplish these functions. We use a MCU STM32 to program the DDS online. The two square waves via 13 and 14 pins are designed to be $90^{\circ}$ phase-shifted, as is shown in Fig .2.

The AD9851 is a highly integrated device that uses advanced DDS technology, coupled with an internal high speed, high performance D/A converter, and comparator, to form a digitally programmable frequency synthesizer and clock generator function.
The square wave generator is referenced to an accurate clock source so that to generate a table frequency and phase-programmable digitized analog output sine wave. This sine wave is internally converted to a square wave for PSD applications.

The phase shifting circuit is embedded in the AD9851 which provides five bits of programmable phase modulation resolution to enable phase shifting of its output in increments of $11.25^{\circ}$. This register can be loaded in parallel or serial mode. A logic high engages functions.

The square wave generator is designed in parallel load mode. The 40-bit input register is loaded using the 8-bit bus of the STM32. W_CLK is used to load the register in five iterations of eight bytes. The rising edge of FQ_UD transfers the contents of the register into the device to be acted upon and resets the word address pointer to W0. Subsequent W CLK rising edges load 8-bit data, starting at W0 and then move the word pointer to the next word. After W0 through W4 are loaded, additional W_CLK edges are ignored until either a RESET is asserted or an FQ UD rising edge resets the address pointer to $\mathrm{W} 0$ in preparation for the next 8-bit load.

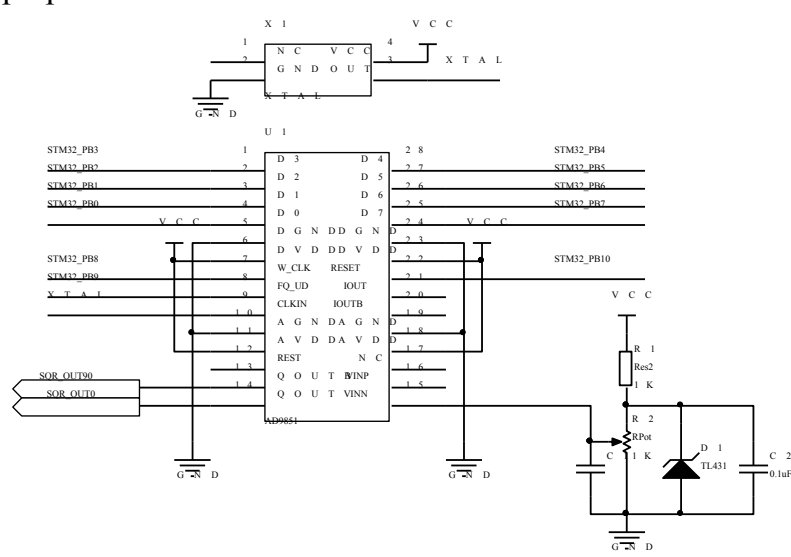

Figure 2. Square wave generator and phase-shifting circuit

\section{The PSD Circuit}

The lock-in amplifier is basically a synchronous demodulator followed by a low-pass filter. An important measure of performance in a lock-in amplifier is the dynamic range of its demodulator. We select AD630 to operate as the PSD, shown as Fig .3.

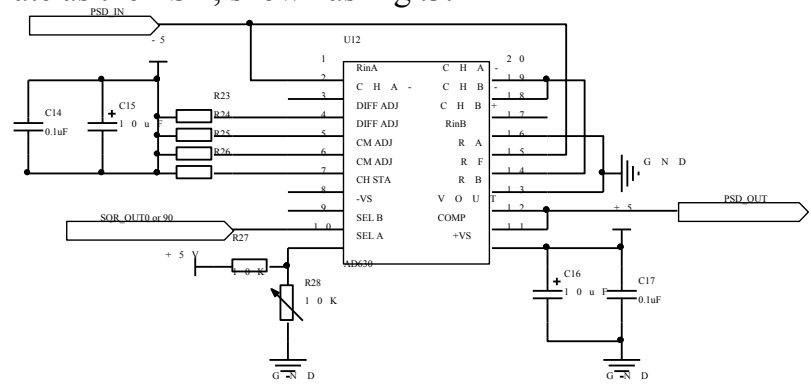

Figure 3. PSD circuit

The AD630 is a high precision balanced modulator which combines a flexible commutating architecture with the accuracy and temperature stability afforded by laser wafer trimmed thin-film resistors. It recovers signal from 
+100 dB noise with $2 \mathrm{MHz}$ Channel Bandwidth. The precision input performance of the AD630 provides more than $100 \mathrm{~dB}$ of signal range.

An important measure of performance in a lock-in amplifier is the dynamic range of its demodulator. The schematic diagram of PSD circuit exhibits the dynamic range of an AD630. The precision input performance of the AD630 provides more than $100 \mathrm{~dB}$ of signal range and its dynamic response permits it to be used with carrier frequencies from $20 \mathrm{~Hz}$ to $20 \mathrm{kHz}$.

The combined modulated signal and interfering noise used for this illustration is similar to the signals often requiring a lock-in amplifier for detection. A more sophisticated low-pass output filter will aid in rejecting wider bandwidth interference.

\section{Programmable Low Pass Filter}

The LIA is basically a synchronous demodulator followed by a low-pass filter. We designed a programmable low pass filter to extract the DC component of the demodulated signal from the PSD, shown in Fig .4.

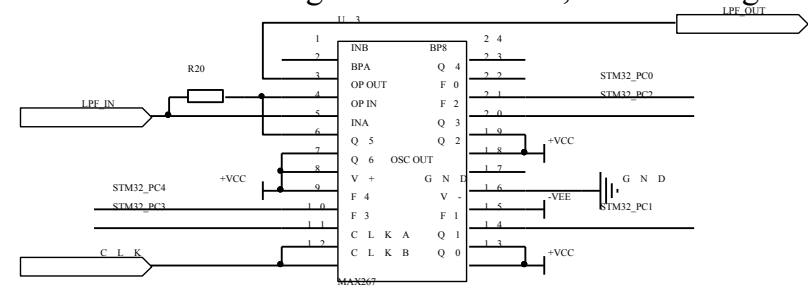

Figure 4. Programmable Low Pass Filter

The programmable LPF is based on MAX267 which is a switched-capacitor active filter and is designed for precision filtering applications. The MAX267 is dedicated to bandpass applications and includes an uncommitted opamp. It includes two second-order filter sections.

The external clock input connects to another square wave generator whose schematic is the similar to Fig .2. The 5-bit programming input pins are connected to MCU.

The MAX267 operates with center frequencies up to $140 \mathrm{kHz}$.by employing lower $f_{C L K} / f_{0}$ ratios. The actual center frequency is a function of the filter's clock rate, shown as follow.

$$
f_{0}=f_{C L K} / 100
$$

MCU program the AD9851 to generate clock with the frequency of $f_{C L K}$. Therefore the center frequency is programed accordingly.

\section{APPLICATION TO WEAK MAGNETIC FIELD MEASUREMENT SYSTEM}

\section{A. Hardware Framwork of the Measurement System}

The LIA is applied to an inducted magnetic field measurement system which is used to detect the location of under grounded pipelines.

The measurement equipment is interfered easily due to the complicated EMI environment. They are often caused by switching operations, thunder-lightning, radiation and harmonic. Moreover, power frequency field is a strong EMI source itself. The anti-interference ability of testing equipment has to be raised by application of LIA.
Fig .5 shows the framework of the measurement system.

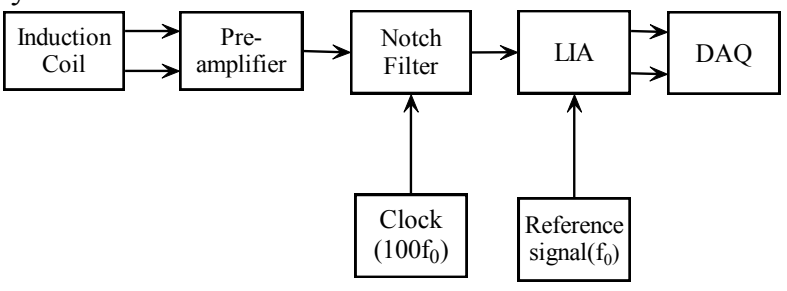

Figure 5. Framwork of the measurement system

In order to measure effectually the surface inducted magnetic of the under-grounded pipeline, it is essential to adopt some technologies of pre-amplifier and notch filter.

According to Faraday electromagnetic induction theorem, the inducted magnetic field can be changed to induction voltage by the induction coil.

The notch filter restrains the high-level $50 \mathrm{~Hz}$ interference in the measuring situation. The frequency of the desired signal is $f_{0}$. So the reference signal's frequency is set to be $f_{0}$ accordingly. And thus the clock is $100 f_{0}$ according to equation(8).

The output of the LNA has two branches. One is the real part while the other is the imaginary part. The DAQ circuit sample the two signals and send the data to the MCU which calculates to obtain the results of the amplitude and the phase according to equation (7).

\section{B. DAQ Circuit}

The DAQ(Data Acquisition) circuit is consist of a MCU STM32F103 and an AD converter AD7193, as shown in Fig. 6.

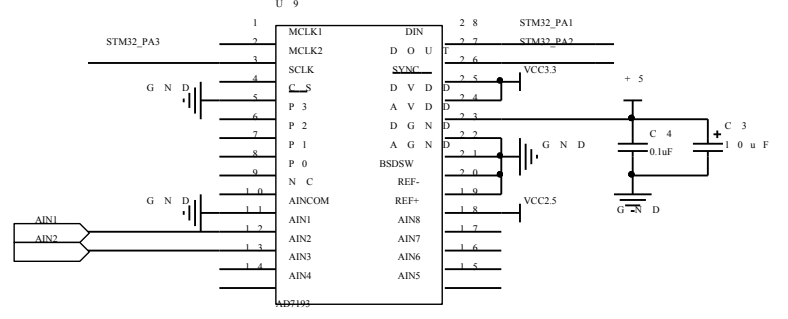

Figure 6. DAQ circuit

The measured two components of the magnetic field are led to two sample channels of the AD7193. The analog power source of the AD shares a same $+5 \mathrm{~V}$ with the LIA, while the digital power source of the $\mathrm{AD}$ is given by the $+3.3 \mathrm{~V}$ of the MCU to avoid additional level translator in the same digital circuit.

For the purpose of increasing SNR, the weak measured voltage signal must be amplified and filtered. These signal process circuit can be simply programed inside the AD7193 which is embedded by a pre-amplifier, a filter and a PGA. The amplification factor can be programed to 1,8 , $16,32,64$, or 128 as needed. The embedded filter shows an excellent noise performance even in a whole data transmission rate. Moreover, AD7193 offers a programmable $50 \mathrm{~Hz} / 60 \mathrm{~Hz}$ notch filter.

An extra voltage reference is designed to connect to AD7193. The reference IC is selected to be LTC6655-2.5. This IC has ultra-low working noise $\left(625 \mathrm{nV}_{\mathrm{p}-\mathrm{p}}\right)$ in the frequent band from $0.1 \mathrm{~Hz}$ to $10 \mathrm{~Hz}$. In the DAQ circuit, the 
power source pin of LTC6655-2.5 is connected to the power source pin of the sensor. Therefore the low frequency noise on power line is minimized. Fig .7 shows the voltage reference circuit.

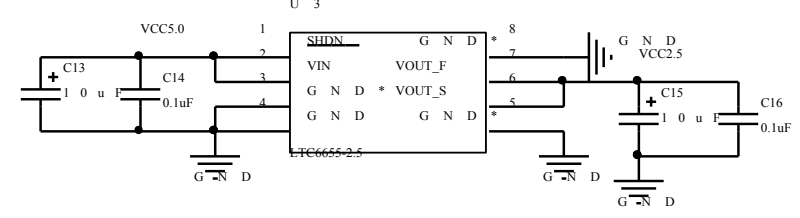

Figure 7. Voltage reference circuit

\section{EXPERIMENT AND TEST}

The schematic design and PCB layout design have been accomplished. The hardware and software debugging have been also performed to make the whole system normal. In the aim of verifying the reliability of the LIA, practical examinations and measurements have been carried out.

Fig .8 shows the main structure of tested grids. The mesh conductors are made copper whose length and width are individually $10 \mathrm{~m}$ and $5 \mathrm{~m}$. The simulation burying depth is $0.9 \mathrm{~m}$

A driving current signal $(2 \mathrm{~A}, 437 \mathrm{~Hz})$ is poured into the grids from the point $\mathrm{A}$ and extracted from the point $\mathrm{B}$ by the back-flow wire. We measure the weak inducted magnetic field over the surface.

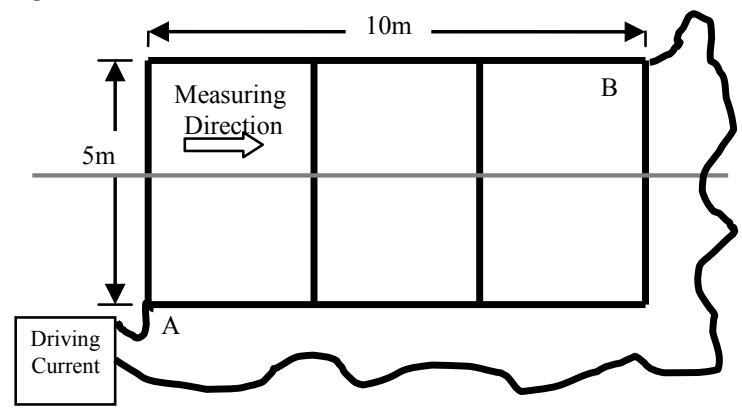

Figure 8. Experiment 1

The voltages of the coil are measured and recorded in horizontal direction with the interval distance of $20 \mathrm{~cm}$. The sampled values are substituted into the equation (7) to get the amplification and phase of the inducted magnetic field at each point. The curve of points vs. amplitudes is drawn as is shown in Fig .9.

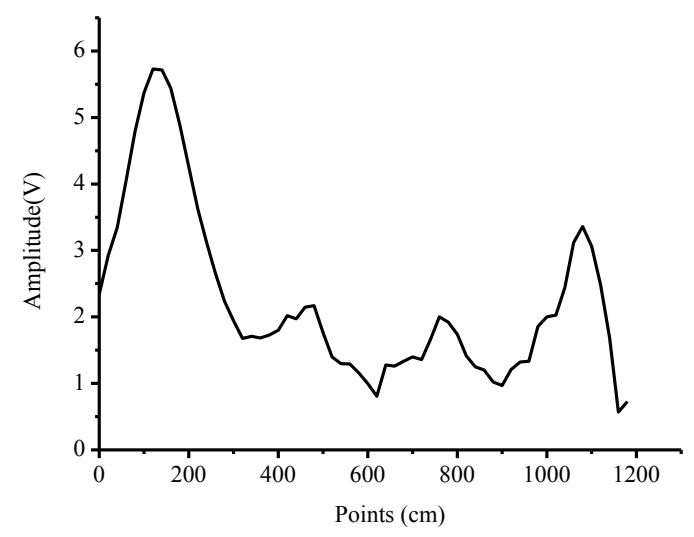

Figure 9. Amplifications of magnetic field

Another test is performed as shown in Fig .10. The driving current signal $(2.2 \mathrm{~A}, 437 \mathrm{~Hz})$ is poured into the grids from the point $\mathrm{C}$ and extracted from the point $\mathrm{C}$.

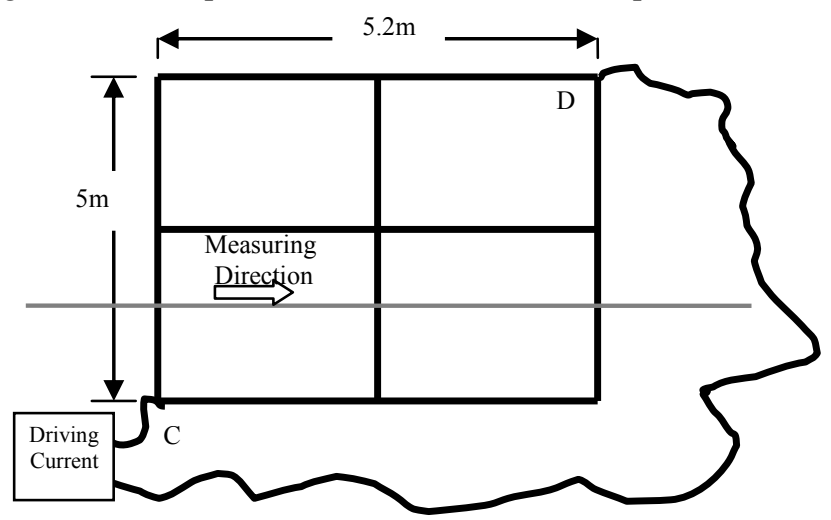

Figure 10. Experiment 2

The curve of points vs. amplitudes is drawn as is shown in Fig .11.

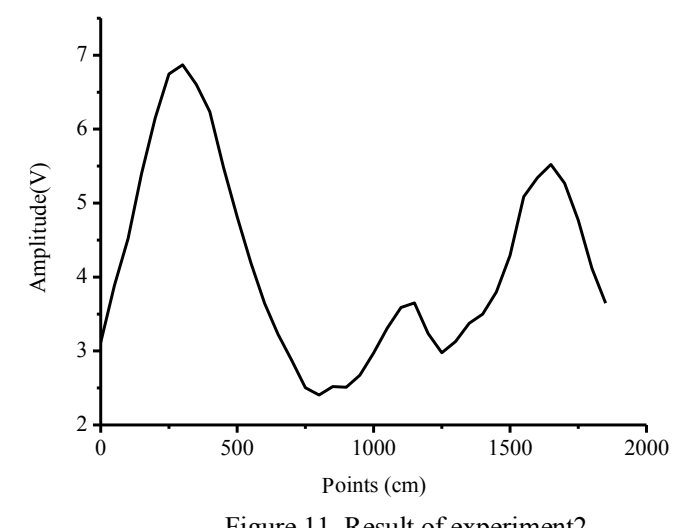

Figure 11. Result of experiment2

From the results of the two experiments, all the peaks of the curves are all corresponding to the locations of the grids which implies the feasibility of the measurement system based on the LIA.

\section{CONCLUSION}

From the above design and applications of LIA, following conclusions can be drawn definitely.

1) A LIA circuit has been designed and debugged to be normal. The LIA involves two PSD branches and programmable LPF.

2) The proposed LIA has been applied to measurement of the surface inducted magnetic field which is weak and in a high-level noise environment. The experimental results show that the LIA is feasible. The measurement system is reliable and can be used in engineering practice.

\section{ACKNOWLEDGMENT}

This work is proudly supported by the National Natural Science Foundation of China (NO. 41474158).

\section{REFERENCES}

[1] L.T. Huang, X.T. Han, J.T. Shi, J.F. Xie, and L. Li, "A study on the weak signal detection system applied to the high field research based on the digital lock-in technique and wavelet filter,", Springer New York, 2013, pp.469-474, doi:10.1007/s10909-012-0719-z. 
[2] R. Takai, S. Uchida, A. Sato, M. Inamori, and Y. Sanada, "Experimental investigation of signal sensing with overlapped FFT based energy detection," Wireless Pers Commun, vol. 77, no. 1, 2014, pp.553-569, doi:10.1007/s11277-013-1522-0.

[3] M. Cunha and C. Richter, "A time-frequency analysis on the impact of climate variability on semi-natural mountain meadows," Ieee T Geosci Remote, vol. 52, no. 10, 2014, pp.6156-6164, doi:10.1109/TGRS.2013.2295321.

[4] J.J. Hudson, M.R. Tarbutt, B.E. Sauer, and E.A. Hinds, "Stochastic multi-channel lock-in detection," New J Phys, vol. 16, 2014, doi:10.1088/1367-2630/16/1/013005.

[5] G. Li, M. Zhou, X. Li, and L. Lin, "Digital lock-in algorithm and parameter settings in multi-channel sensor signal detection," Measurement: Journal of the International Measurement Confederation, vol. 46, no. 8, 2013, pp.2519-2524, doi:10.1016/j.measurement.2013.05.014.

[6] [6]R. Augulis and D. Zigmantas, "Two-dimensional electronic spectroscopy with double modulation lock-in detection: Enhancement of sensitivity and noise resistance," Opt Express, vol. 19, no. 14, 2011, pp.13126-13133, doi:10.1364/OE.19.013126.
[7] Y. Liu, W. Quan and T. Wang, "Research on methods for weak signal detection of atomic magnetometers,", IEEE Computer Society, 2012, pp.286-290, doi:10.1109/ISICT.2012.6291619.

[8] J. Aguirre, N. Medrano, B. Calvo, and S. Celma, "Lock-in amplifier for portable sensing systems," Electron Lett, vol. 47, no. 21, 2011, pp.1172-1173, doi:10.1049/el.2011.2472.

[9] P.M. Maya-Hernandez, L.C. Alvarez-Simon, M.T. Sanz-Pascual, and B. Calvo-Lopez, "An integrated low-power lock-in amplifier and its application to gas detection," Sensors (Switzerland), vol. 14, no. 9, 2014, pp.15880-15899, doi:10.3390/s140915880.

[10] F. Hoehne, L. Dreher, J. Behrends, M. Fehr, H. Huebl, K. Lips, A. Schnegg, M. Suckert, M. Stutzmann, and M.S. Brandt, "Lock-in detection for pulsed electrically detected magnetic resonance," Rev Sci Instrum, vol. 83, no. 4, 2012, doi:10.1063/1.4704837.

[11] A. De Marcellis, G. Ferri, A. D'Amico, C. Di Natale, and E. Martinelli, "A fully-analog lock-in amplifier with automatic phase alignment for accurate measurements of ppb gas concentrations," IEEE Sensors Journal, vol. 12, no. 5, 2012, pp.1377-1383, doi:10.1109/JSEN.2011.2172602. 